\title{
Formation and Evolution of Galactic Intermediate/Low-Mass X-ray Binaries
}

\author{
Yong Shao ${ }^{1,2}$ and Xiang-Dong $\mathrm{Li}^{1,2}$ \\ ${ }^{1}$ Department of Astronomy, Nanjing University, Nanjing 210093, China \\ ${ }^{2}$ Key laboratory of Modern Astronomy and Astrophysics (Nanjing University), Ministry of \\ Education, Nanjing 210093, China \\ lixd@nju.edu.cn
}

\begin{abstract}
We investigate the formation and evolutionary sequences of Galactic intermediate- and low-mass X-ray binaries (I/LMXBs) by combining binary population synthesis (BPS) and detailed stellar evolutionary calculations. Using an updated BPS code we compute the evolution of massive binaries that leads to the formation of incipient I/LMXBs, and present their distribution in the initial donor mass vs. initial orbital period diagram. We then follow the evolution of I/LMXBs until the formation of binary millisecond pulsars (BMSPs). We find that the birthrate of the I/LMXB population is in the range of $9 \times 10^{-6}-3.4 \times 10^{-5} \mathrm{yr}^{-1}$, compatible with that of BMSPs which are thought to descend from I/LMXBs. We show that during the evolution of I/LMXBs they are likely to be observed as relatively compact binaries with orbital periods $\lesssim$ 1 day and donor masses $\lesssim 0.3 M_{\odot}$. The resultant BMSPs have orbital periods ranging from less than 1 day to a few hundred days. These features are consistent with observations of LMXBs and BMSPs. We also confirm the discrepancies between theoretical predications and observations mentioned in the literature, that is, the theoretical average mass transfer rates $\left(\sim 10^{-10} M_{\odot} \mathrm{yr}^{-1}\right)$ of LMXBs are considerably lower than observed, and the number of BMSPs with orbital periods $\sim 0.1-10$ day is severely underestimated. These discrepancies imply that something is missing in the modeling of LMXBs, which is likely to be related to the mechanisms of the orbital angular momentum loss.
\end{abstract}

Subject headings: binaries: general - X-ray: binaries - stars: neutron - stars: evolution 


\section{Introduction}

Low-mass X-ray binaries (LMXBs) consist of an accreting compact star, either a black hole or a neutron star (NS) and a low-mass $\left(\lesssim 1 M_{\odot}\right)$ donor star, in which mass transfer proceeds via Roche-lobe overflow (RLOF). There are about 200 LMXBs in the Galaxy (Liu, van Paradijs, \& van den Heuvel 2007). The formation of black hole LMXBs is still a controversial topic ( $\operatorname{Li} 2015$, for a review), and will be discussed elsewhere. Here we focus on the formation and evolution of LMXBs with a NS. The mainstream idea is that they either have an initially low-mass secondary, or descend from systems with initially intermediate-mass secondaries, i.e., intermediate-mass X-ray binaries (IMXBs; Podsiadlowski \& Rappaport 2000; Kolb et al. 2000; Tauris et al. 2000; Podsiadlowski et al. 2002). Considering the fact that NSs must have formed from massive stars, and current LMXBs usually reside in relatively compact binary orbits (with periods $P_{\text {orb }} \lesssim 10$ days; see Liu, van Paradijs, \& van den Heuvel 2007; Wu et al. 2010; Revnivtsev et al. 2011), the progenitors of these systems should generally have experienced common envelope (CE) evolution (Paczynski 1976). During the CE phase and the subsequent supernova (SN) explosion, only a small part of the binaries can survive as I/LMXBs (Pfahl et al. 2003). For reviews on the formation and evolution of I/LMXBs, see Bhattacharya \& van den Heuvel (1991) and Tauris \& van den Heuvel (2006).

The stability of the mass transfer in I/LMXBs depends on the mass ratio, the orbital period, and the mass and angular momentum loss (AML; Soberman et al. 1997). Dynamically unstable mass transfer leads to a (second) CE evolution, with the NS spiraling into the envelope of the donor star. If the binary survives without merging, it becomes a compact binary with a helium (He) star around the NS. The subsequent mass transfer from the He star may lead to the formation of a partially recycled pulsar with a CO white dwarf (WD) companion (van den Heuvel \& Taam 1984, Chen et al. 2011). For those binaries avoiding the CE phase, Pylyser \& Savonije (1988, 1989) pointed out that there exists a bifurcation period $\left(P_{\text {bif }}\right)$ for the initial orbital period, which separates I/LMXBs into converging or diverging systems. The existence of this bifurcation period is due to the balance between the orbital expansion caused by nuclear evolution of the donor star and the orbital shrinking caused by AML. Binaries with $P_{\text {orb }}<P_{\text {bif }}$ continue to contract along the cataclysmic variable (CV)-like or ultra-compact X-ray binary (UCXB) evolutionary tracks, while those with $P_{\text {orb }}>P_{\text {bif }}$ will become relatively wide binaries containing a recycled NS and a He or CO WD (Rappaport et al. 1995; Kalogera \& Webbink 1996; King \& Ritter 1999; Tauris \& Savonije 1999; Tauris et al. 2000; Podsiadlowski \& Rappaport 2000; Kolb et al. 2000; Li 2002; Deloye 2008; De

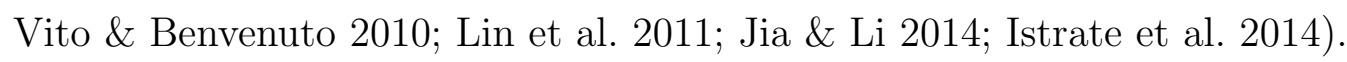

Podsiadlowski et al. (2002) made a systematic investigation of the evolution of I/LMXBs by using a Henyey-type stellar evolution code to calculate 100 binary evolution sequences. 
In that work, the initial mass of the NS was fixed as $1.4 M_{\odot}$, the initial mass of the donor ranged from $0.6-7 M_{\odot}$, and the initial orbital period went from $4 \mathrm{hr}$ to 100 days. While the calculated results showed a variety of evolutionary channels which may explain the large diversity in observed LMXBs, they also indicated a number of remaining problems, in particular the low median X-ray luminosities as compared to those of observed LMXBs.

Pfahl et al. (2003) firstly combined the binary population synthesis (BPS) method with detailed stellar evolutionary sequences of Podsiadlowski et al. (2002) to follow the evolution of a population of I/LMXBs. For early Cases B and C mass transfer ${ }^{1}$ they adopted a fixed value of the critical ratio of the donor mass and the NS mass $\left(q_{\mathrm{c}}=2\right)$ to determine whether the mass transfer is stable. Late Cases B and C mass transfer was always assumed to be dynamically unstable followed by a CE phase. When dealing with CE evolution, they adopted constant values of the binding energy parameter $\lambda=0.1,0.3$ and 0.5 , which parameterizes the structure of the donor's envelope. Their calculations demonstrated that incipient IMXBs outnumber incipient LMXBs typically by a factor of $\gtrsim 5$. It was also found that rather large values of $\lambda \sim 0.5$ are required to yield the I/LMXB birthrates that are consistent with the BMSP birthrates, but such values of $\lambda$ would lead to an overproduction of luminous LMXBs in the Galaxy. There were still large discrepancies between the orbital period distributions of modeled and observed binary millisecond pulsars (BMSPs). A possible solution to the above problems was to reduce the mean X-ray lifetime of LMXBs by a factor of $\gtrsim 100$.

In this work, we revisit the formation and evolution of Galactic I/LMXBs. Similar as Pfahl et al. (2003), we perform a systematic evolutionary study of I/LMXBs, combining BPS with detailed binary evolutionary sequences. To follow the formation and evolution of I/LMXBs, we take into account updated theoretical results and techniques on binary evolution achieved during the last ten years. We use the rapid binary evolution algorithm developed by Hurley et al. (2002) for BPS calculation, which enables modeling of a large number of complex binary systems to obtain the incipient population of I/LMXBs, and an updated version of the Eggleton (1971, 1972) code to calculate the detailed evolution of individual I/LMXBs. With them we can follow the evolutionary tracks of the I/LMXB population from the incipient stage of a detached binary containing a newborn NS to the RLOF phase, and finally to the remnant state with a BMSP. The parameter distributions of I/LMXBs and BMSPs can be clearly displayed, and compared with observations.

The most important and difficult to understand processes during binary evolution are

\footnotetext{
${ }^{1}$ Cases A, B and C means that the donor stars are in core hydrogen burning, shell hydrogen burning, and after exhaustion of core helium burning stages, respectively.
} 
unstable mass transfer and CE evolution (Ivanova et al. 2013, for a recent review). In the BPS calculations, instead of using the empirical relations for the critical mass ratios, we employ the numerically calculated results of Shao \& Li (2014) to judge the stability of mass transfer in a primordial binary, and the parameter space obtained by Shao \& Li (2012b, hereafter Paper I) to determine whether I/LMXBs can successfully evolve to be binary

pulsars. We also adopt the calculated values of $\lambda$ given by $\mathrm{Xu} \& \mathrm{Li}(2010)$ to calculate the orbital change during the CE phase, which depend on the evolutionary state of the donor. Other improvement will be mentioned below. Although these updated parameters are still subject to large uncertainties, they provide physically more reasonable and realistic input as compared to previous BPS studies.

This paper is organized as follows. In Section 2 we describe the methods used in this paper. We present the detailed results derived from our calculations and discuss their implications in Section 3. We conclude in Section 4.

\section{Methods and Calculations}

\subsection{Formation of Incipient I/LMXBs}

We use the BPS code developed by Hurley et al. (2002) and modified by Kiel \& Hurley (2006) to generate the incipient I/LMXB population. This code, based on single star evolution with a series of analytic formulae (Hurley et al. 2000), includes standard assumptions for the population of massive primordial binaries and analytic prescriptions to describe binary interactions, mass transfer and SN explosions. We have updated the code in various aspects, in particular the formation and evolutionary processes of compact objects. Some key points are described below (see Shao \& Li 2014, for more details).

The stability of mass transfer is determined by the change of the donor's radius with respect to its $\mathrm{RL}$ radius. Usually a critical mass ratio $q_{\mathrm{c}}(=$ donor mass/accretor mass) is used as a criterion: if the mass ratio is larger than $q_{\mathrm{c}}$ at the onset of RLOF, the mass transfer is unstable and results in a CE evolution; otherwise the mass transfer proceeds stably on a nuclear or thermal timescale. In previous studies, the values of $q_{\mathrm{c}}$ were usually estimated by only comparing the mass-radius exponents for the donor stars, which describe the response of the star and the RL to mass loss (Hjellming \& Webbink 1987). However, it is well known that mass accretion can spin up the accretor ( (de Mink et al. 2009). Rapid mass accretion can drive the accreting star out of thermal equilibrium and cause it to expand, and this expansion may even cause the accretor to fill its own RL, leading to the formation of a contact binary (Nelson \& Eggleton 2001). Shao \& 
Li (2014) numerically calculated the values of $q_{\mathrm{c}}$ for a grid of binaries with different values of component masses and orbital periods, considering the responses of both the donor to mass loss and the accretor to mass accretion. Since the stellar expansion strongly depends on the accretion rate, they constructed three models with different assumed mass accretion histories. In Model I, the mass accretion rate depends on the rotational velocity of the accretor. Since a small amount of transferred material is able to spin it up to be critically rotating (Packet 1981), there is actually very small mass accreted in this case. In Model II, it is assumed that half of the transferred mass is accreted and the other half is ejected out of binary system. In model III, the mass transfer is assumed to be almost conservative. The obtained values of $q_{\mathrm{c}}$ vary with the orbital period, and can reach $\sim 6$ in Model I, but are generally less than $\sim 2.5$ and $\sim 2.2$ in Models II and III, respectively.

For unstable mass transfer, we use the standard energy conservation equation (Webbink 1984) to describe the subsequent CE evolution,

$$
\alpha_{\mathrm{CE}}\left(\frac{G M_{1, \mathrm{f}} M_{2}}{2 a_{\mathrm{f}}}-\frac{G M_{1, \mathrm{i}} M_{2}}{2 a_{\mathrm{i}}}\right)=\frac{G M_{1, \mathrm{i}} M_{1, \mathrm{env}}}{\lambda R_{1, \mathrm{~L}}},
$$

where the indices 1 and 2 denote the primary and the secondary, and $\mathrm{i}$ and $\mathrm{f}$ the initial and final values, respectively; $M_{1, \text { env }}$ is the mass of the primary's envelope that is ejected out of the binary during the $\mathrm{CE}$ phase, $R_{1, \mathrm{~L}}$ is the $\mathrm{RL}$ radius of the primary at the onset of RLOF, and $\alpha_{\mathrm{CE}}$ is the CE efficiency with which the orbital energy is used to unbind the stellar envelope. It has been pointed out that the internal energy within the envelope can also contribute to envelope ejection, and thus modify the values of $\lambda$ (e.g., de Kool 1990; Dewi \& Tauris 2000; Podsiadlowski et al. 2003). We employ the values of $\lambda$ calculated by $\mathrm{Xu} \& \mathrm{Li}(2010)$ for a range of massive and intermediate-mass stars at various evolutionary stages, and take $\alpha_{\mathrm{CE}}=1.0$ in the calculations.

We assume that NSs are born from either core-collapse or electron-capture SNe, depending on the masses of the progenitor stars. We follow the criterion suggested by Belczynski et al. (2008) and Fryer et al. (2012) to distinguish them. The He core mass at the base of asymptotic giant branch is used to decide the formation of various CO cores. (1) If the He core mass is less than $1.83 M_{\odot}$, the star forms a degenerate $\mathrm{CO}$ core and ends up forming a CO WD. (2) If the core is more massive than $2.25 M_{\odot}$, the star ultimately forms a FeNi core and collapses to a NS. (3) Stars with core masses between $1.83 M_{\odot}$ and $2.25 M_{\odot}$ form partially degenerate $\mathrm{ONe}$ cores. If in subsequent evolution the core mass increases to $1.38 M_{\odot}$, then the core collapses to a NS due to electron capture; otherwise the star leaves an ONe WD. Under these prescriptions, for single stars, the stellar masses for core-collapse and electroncapture SNe are in the range of $8.3-20 M_{\odot}$, and $7.6-8.3 M_{\odot}$, respectively (Belczynski et al. 2008). The masse interval for electron-capture SNe is in rough accordance with Woosley \& 
Heger (2015). It depends on the assumptions for mass loss and the dredge-up process in stellar evolution (e.g., Siess 2006; Poelarends et al. 1988; Jones et al. 2014), and is blurred by mass transfer in binaries (Hurley et al. 2002). The newborn NSs are assumed to receive a kick due to asymmetric SN explosions, and we utilize a Maxwellian distribution for the kick velocity,

$$
p\left(v_{\mathrm{k}}\right)=\sqrt{\frac{2}{\pi}}\left(\frac{v_{\mathrm{k}}^{2}}{\sigma_{\mathrm{k}}^{2}}\right) e^{-v_{\mathrm{k}}^{2} / 2 \sigma_{\mathrm{k}}^{2}},
$$

assuming that the directions of the kicks are isotropically distributed. We adopt $\sigma_{\mathrm{k}}=$ $265 \mathrm{~km} \mathrm{~s}^{-1}$ (Hobbs et al. 2005) and $50 \mathrm{~km} \mathrm{~s}^{-1}$ (Dessart et al. 2006) for NSs formed from core-collapse and electron-capture $\mathrm{SNe}$, respectively.

The initial parameters in our BPS calculation are taken as follows. All the binary orbits are assumed to be circular due to tidal interactions (Hurley et al. 2002). The mass $M_{1}$ of the primary is in the range of $3-30 M_{\odot}$, following the initial mass function of Kroupa et al. (1993) The initial mass ratio $M_{2} / M_{1}$ has a uniform distribution between 0 and 1 , but only binaries with the secondary mass $M_{2}$ between $0.1 M_{\odot}$ and $8 M_{\odot}$ are chosen. The logarithm of the orbital separation $a$ is uniformly distributed, lying between $3 R_{\odot}$ and $10^{4} R_{\odot}$. We adopt solar metallicity, and assume a constant star formation rate of $5 M_{\odot} \mathrm{yr}^{-1}$ over the past 12 Gyr (Smith et al. 1978). Robitaille \& Whitney (2010) obtained a smaller star formation rate of $\sim 1 M_{\odot} \mathrm{yr}^{-1}$ derived from Spitzer detected pre-main-sequence stars. If we adopt the latter value, the predicted number of Galactic I/LMXBs will be reduced by several times, but the features of their distribution will not change.

In Fig. 1, we plot the birthrates of incipient I/LMXBs as a function of the donor mass $M_{\mathrm{d}}$ (in the range of $0.3-6 M_{\odot}$ ). In the left and right panels the NSs are assumed to form from core-collapse and electron-capture SNe, respectively. The green, red and black curves correspond to the results with the $q_{\mathrm{c}}$ values from Models I, II and III, respectively. It is clearly seen that, in both core-collapse and electron-capture SN channels, IMXBs dominate the whole population as expected. Although core-collapse SN-IMXBs in Model I have a birthrate higher than in other models by $\lesssim 50 \%$, the overall birthrate distributions do not show a remarkable difference. Thus we only take the calculated birthrates in Model II in the following study, not only because it is intermediate between the other two more extreme ones, but also because the corresponding results can better match the observed distribution of Be/X-ray binaries (Shao \& Li 2014).

In Fig. 2 we plot the distributions of incipient I/LMXBs in the orbital period - donor

\footnotetext{
${ }^{2}$ In the rapid SN mechanism of Fryer et al. (2012), the upper limit for the masses of the NS progenitors in binaries is $28 M_{\odot}$.
} 
mass plane (left panel: the core-collapse SN channel; right panel: the electron-capture SN channel). After SNe the binary orbits become eccentric due to mass loss and the kicks imparted on the NSs. Here we assume that they are quickly circularized by tidal torques with the orbital AM conserved. The corresponding orbital separation is then set to be $a_{\mathrm{SN}}(1-e)$, where $a_{\mathrm{SN}}$ is the semimajor axis after the SNe. Also shown are the histogram distributions of the birthrates, same as the red curves in Fig. 1. In the core-collapse SN channel, the NS progenitors masses are $8.6-28 M_{\odot}$, the resultant I/LMXBs have orbital periods $P_{\text {orb }} \lesssim 100$ days, and most of them have $P_{\text {orb }}<$ a few days. The I/LMXBs birthrate roughly increases with the donor mass, because systems with lower-mass companions are more likely to merge during the CE phase (see also Pfahl et al. 2003). In the electron-capture SN channel, the masses of the NS progenitors lie in the range $7.6-8.6 M_{\odot}$. The primordial binaries should have orbital periods longer than $\sim 1000$ days, otherwise the mass transfer starts too early so that a WD rather a NS is produced. The binding energies for such stars are relatively small, with the values of $\lambda$ greater than unity when on asymptotic giant branch (Dewi \& Tauris 2000; Xu \& Li 2010). So the resultant I/LMXBs have orbital periods in a wide range $\sim 0.2-1000$ days, but the majority are relatively long period ( $P_{\text {orb }}>10$ days) systems. The I/LMXB birthrate slightly decreases with increasing donor mass. The reason is that, for relatively massive companion stars, the orbital periods after CE are usually long because there are sufficient orbital kinetic energies. The second mass transfer may not occur, so the primaries undergo core-collapse $\mathrm{SNe}$, which can readily disrupt the wide binaries. Finally there are no binaries with $P_{\text {orb }} \gtrsim 1$ day and $M_{\mathrm{d}} \lesssim 1 M_{\odot}$, because magnetic braking $(\mathrm{MB})$ and gravitational radiation (GR) cannot drive these systems into contact within Hubble time.

\subsection{Evolution of I/LMXBs}

Based on the results in last subsection we then employ an updated version of the Eggleton $(1971,1972)$ code to calculate the evolution of I/LMXBs. Before doing this, we first select the binaries in which mass transfer is stable so that they can evolve to be binary pulsars. Super-Edddinton accretion onto NSs in IMXBs may lead to delayed dynamical instability, and eventually $\mathrm{CE}$ evolution. However, there are observational and theoretical hints for mass loss from the accreting NSs that can stabilize the mass transfer (King \& Ritter 1999 ; Podsiadlowski \& Rappaport 2000, and references therein). Tauris et al. (2000) surveyed the allowed parameter $\left(M_{\mathrm{d}}-P_{\text {orb }}\right)$ space for stable mass transfer, and showed that the donor masses $M_{\mathrm{d}}$ can be up to $5 M_{\odot}$ for a $1.3 M_{\odot}$ NS. Observations of high-mass X-ray binaries, in which the NSs have accreted very small mass, show that the initial masses of NSs range from $\sim 1 M_{\odot}$ for $4 \mathrm{U} 1538-52$ to $\sim 1.8 M_{\odot}$ for Vela X-1 (Rawls et al. 2011). In Paper I we extended the calculation by considering the NS masses $M_{\mathrm{NS}}$ in the range of $1.0-1.8 M_{\odot}$. The 
results are used to compare with the obtained incipient I/LMXB distribution in Fig. 3 (binaries formed from both core-collapse and electron-capture SN channels are included). The left and right panels are for NSs with mass $1.0 M_{\odot}$ and $1.8 M_{\odot}$, respectively. The binaries distributed in the regions confined by the thick grey curves can stably evolve without entering the CE phase, and are selected for further investigation. Their corresponding birthrates are shown with the blue curves, totally ranging from $9 \times 10^{-6}$ to $3.4 \times 10^{-5} \mathrm{yr}^{-1}$. Figure 4 demonstrates the distributions of the birthrates for these systems as a function of the initial donor masses and the orbital periods. Here the bin size of the donor mass is $0.2 M_{\odot}$ and the orbital period (in units of days) changes logarithmically in steps of 0.2 .

We calculate the evolution of the selected I/LMXBs in a similar way as in Paper I, taking into account AML caused by MB (Verbunt \& Zwaan 1981; Rappaport et al. 1983) and GR (Landau \& Lifshitz 1959; Faulkner 1971). The accretion rate of a NS is assumed to be limited by the Eddington accretion rate, and we adopt the isotropic re-emission model, assuming that the excess material leaves the binary in the form of isotropic winds from the NS, carrying off the $\mathrm{NS}^{\prime}$ s specific orbital AM.

Figures 5 and 6 present two example evolutionary tracks. In Fig. 5 the initial parameters are $M_{\mathrm{NS}}=1.8 M_{\odot}, M_{\mathrm{d}}=1.0 M_{\odot}$ and $P_{\mathrm{orb}}=1.0$ day. The left, middle, and right panels depict the evolution of the orbital period $P_{\text {orb }}$ with the age, the evolution of the donor radius $R_{\mathrm{d}}$ and the mass transfer rate $\left|\dot{M}_{\mathrm{d}}\right|$ with the donor mass $M_{\mathrm{d}}$, respectively. The donor starts to fill its RL and initiate (Case A) mass transfer at $t=2.23$ Gyr when $P_{\text {orb }}=0.33$ day, mainly driven by MB. The mass transfer rate first goes up to $\sim 2 \times 10^{-9} M_{\odot} \mathrm{yr}^{-1}$, then decreases gradually as the orbital period reduces to $\sim 0.1$ day. Meanwhile, the donor mass decreases to be less than $0.3 M_{\odot}$, and the donor becomes fully convective, so MB stops working. The evolution afterwards is solely driven by GR, and the mass transfer rate drops down to $\sim 10^{-10} M_{\odot} \mathrm{yr}^{-1}$. At the end of the evolution (the age of $\sim 6 \mathrm{Gyr}$ ), the donor mass and the orbital period are reduced to $\sim 0.08 M_{\odot}$ and $\sim 0.06$ day respectively. The duration of the mass transfer is about 3.77 Gyr.

In Fig. 6 we show the evolutionary path with the same component masses but $P_{\text {orb }}=6.3$ days. The mass transfer starts at the age of $11.9 \mathrm{Gyr}$, when the donor evolves to become a subgiant. During this Case B mass transfer, the orbital period increases all the time, up to 73 days. The mass transfer rate varies in the range $10^{-9}-10^{-8} M_{\odot} \mathrm{yr}^{-1}$ (the gap in the mass transfer rate is caused by the so-called "bump-related" detachment; D'Antona et al. 2006). The binary finally leaves a NS with a He WD with mass of $0.31 M_{\odot}$. The mass transfer liftime is about 0.14 Gyr.

The above examples suggest that, given the same donor star, the longer the initial orbital periods, the higher the averaged mass transfer rates, and the shorter the X-ray 
lifetimes (Webbink et al. 1983; King 1988). This strongly influences the characteristics of observable LMXBs.

\section{Results and Discussion}

\subsection{Orbital periods, accretion rates and donor star masses of Galactic I/LMXBs}

According to the calculated evolutionary sequences of the I/LMXBs, we plot their number distribution in the orbital period - mass transfer rate $\left(P_{\text {orb }}-\left|\dot{M}_{\mathrm{d}}\right|\right)$ plane (Fig. 7). The left and right panels correspond to the results with a $1.0 M_{\odot}$ and $1.8 M_{\odot} \mathrm{NS}$, respectively. Each panel contains $45 \times 64$ matrix elements, in which $P_{\text {orb }}$ changes logarithmically from 0.0316 day to 1000 days with steps of 0.1 , and $\left|\dot{M}_{\mathrm{d}}\right|$ from $10^{-12} M_{\odot} \mathrm{yr}^{-1}$ to $10^{-4} M_{\odot} \mathrm{yr}^{-1}$ with steps of 0.125 . The color reflects the number of I/LMXBs in the corresponding matrix element by accumulating the product of the birthrates of X-ray binaries passing through it with the time-span.

A comparison of Figs. 4 and 7 clearly demonstrates the change in the orbital periods during the evolution of I/LMXBs. As we have already seen from Figs. 5 and 6, in X-ray binaries with initially long orbital periods, the donors have evolved to be (sub)giant stars at the onset of mass transfer, and the high mass transfer rates imply a short X-ray lifetime; in short-period X-ray binaries, the donors are still on main-sequence during mass transfer, so the systems have a long-lasting mass transfer phase. This difference in the evolutionary time leads to different number distribution in Fig. 7, which explains why most I/LMXBs tend to have relatively short orbital periods $(\lesssim 1$ day). Another factor that influences the number distribution of LMXBs is their transient behavior. The general idea is that, if the mass transfer rate is smaller than a critical mass transfer rate for a given orbital period, the accretion disk is likely subject to thermal and viscous instability, and the LMXB may appear as a transient X-ray source (Lasota 2001). In LMXBs irradiation from the NS may help stabilize the disk to some extent by increasing the surface temperature van Paradijs 1996; King et al. 1997; Lasota 2001; Ritter 2008). The critical mass transfer rate for the disk instability is given by (Dubus et al. 1999),

$$
\dot{M}_{\text {cr }} \simeq 3.2 \times 10^{-9}\left(\frac{M_{\mathrm{NS}}}{1.4 M_{\odot}}\right)^{0.5}\left(\frac{M_{\mathrm{d}}}{1.0 M_{\odot}}\right)^{-0.2}\left(\frac{P_{\mathrm{orb}}}{1.0 \mathrm{~d}}\right)^{1.4} M_{\odot} \mathrm{yr}^{-1}
$$

and plotted with the red dashed line. We assume that LMXBs under this line experience short outbursts separated by long quiescent intervals. The majority of long-period LMXBs 
are transient sources spending most of their time in quiescence ${ }^{3}$. These results are consistent with Zhu et al. (2012), who showed that more than 90\% persistent NS LMXBs have mainsequence donors and orbital periods between $\sim 1-100$ hours. Comparing the two panels of Fig. 7 also shows that, LMXBs with a heavier NS have a larger coverage in the parameter space, since mass transfer in these binaries are more likely to be stable (see Fig. 3).

In Fig. 7 we also plot Galactic LMXBs with known orbital periods and accretion rates. Here the observational data are taken from Ritter's LMXB catalogue (Ritter \& Kolb 2003) and from Revnivtsev et al. (2011) and Coriat et al. (2012). The filled circles and triangles represent persistent and transient LMXBs, respectively 5. It is clearly seen that most LMXBs are compact systems except two sources: Cyg $\mathrm{X}-2$ with a $\sim 0.6 M_{\odot}$ companion star in a 9.84 day orbit (Casares et al. 1998; Orosz \& Kuulkers 1999), and GX13+1 (shown with the star symbol), which has the longest known orbital period (of 601.7 hours) for a Galactic NS LMXB powered by RLOF (Bandyopadhyay et al. 1999).

Figure 8 depicts the distributions of the masses (left panel) and effective temperatures (right panel) of the donor stars. The upper and lower panels are for persistent and transient LMXBs, and the black and red lines correspond to $1 M_{\odot}$ and $1.8 M_{\odot} \mathrm{NSs}$, respectively. It is seen that LMXBs dominate the population at the current epoch, because the initial phase of thermal-timescale mass transfer in IMXBs, where a large fraction of the secondary mass is removed, is relatively short-lived. The donor masses in LMXBs cluster around $0.2-0.3 M_{\odot}$. The donors' temperatures are distributed in the range of $\sim 2500-6300 \mathrm{~K}$.

Figure 9 presents a more detailed comparison of the modeled orbital period (left panel) and accretion rate (right panel) distributions (in red lines) with observations (in black lines). The upper and lower panels are for $1.0 M_{\odot}$ and $1.8 M_{\odot}$ NSs, respectively. The numbers for observed sources are amplified by a factor of 500. Although there seems broad agreement between the measured and predicted orbital periods, there exist a systematic discrepancy in the accretion rates, i.e., the calculated average accretion rates $\left(\sim 10^{-10} M_{\odot} \mathrm{yr}^{-1}\right)$ are lower than derived from observations by about an order of magnitude.

To quantitatively compare the univariate distributions of the calculated and observed

\footnotetext{
${ }^{3}$ It is interesting to note that all accreting X-ray MSPs have orbital periods less than 19 hours, whereas radio MSPs are found up to $P_{\text {orb }} \sim 200$ days. This puzzle can be partly understood by the transient behavior of X-ray MSPs (Patruno \& Watts 2012 , and references therein).

${ }^{4}$ http://www.mpa-garching.mpg.de/RKcat/

${ }^{5}$ When estimating the average accretion rates for transient LMXBs, Coriat et al. (2012) ignored the possibility of accretion in quiescence and mass loss through winds and jets, so the estimates should be considered as lower limits to the actual mass transfer rates.
} 
quantities, we use Kolmogorov-Smirnov test to assist inference on their consistency (Feigelson \& Babu 2012). The cumulative distribution functions (CDFs) for the calculated and measured orbital periods of the I/LMXBs are expressed as $F\left(P_{\text {orb }}\right)$ and $F_{0}\left(P_{\text {orb }}\right)$, respectively. We want to test the null hypothesis that $F\left(P_{\text {orb }}\right)=F_{0}\left(P_{\text {orb }}\right)$ for all $P_{\text {orb }}$, against

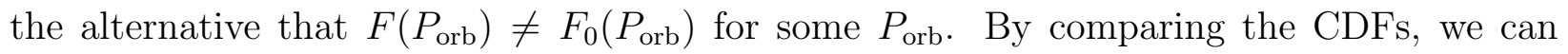
measure the maximum distance $M_{\mathrm{KS}}=\sqrt{\frac{m n}{m+n}} \max \left|F\left(P_{\text {orb }}\right)-F_{0}\left(P_{\text {orb }}\right)\right|$, where $m$ and $n$ are the numbers of X-ray binaries in the calculation and the observation, respectively. Since $m \gg n, M_{\mathrm{KS}} \simeq \sqrt{n} \max \left|F\left(P_{\text {orb }}\right)-F_{0}\left(P_{\text {orb }}\right)\right|$. At a given significance level $\alpha=0.05$, there exists a critical value $M_{\mathrm{KS}}^{\mathrm{cr}} \simeq 1.33$, and $M_{\mathrm{KS}}>M_{\mathrm{KS}}^{\mathrm{cr}}$ allows rejection of the null hypothesis, indicating that the two distributions are significantly different. Figure 10 shows the CDFs for the orbital period (left panel) and the accretion rate (right panel) of the I/LMXBs. The black, red and green curves represent the calculated CDFs with the NS mass of $1.0 M_{\odot}$ and $1.8 M_{\odot}$, and the observed CDF, respectively. For the orbital period distribution, we obtain $M_{\mathrm{KS}}=2.62$ and 1.15 with a $1.0 M_{\odot}$ and $1.8 M_{\odot} \mathrm{NS}$, respectively; while for the accretion rate distribution, $M_{\mathrm{KS}}=4.1$ and 3.4, suggesting that the difference is significant.

We note that similar conclusions were also reached by Podsiadlowski et al. (2002) and Pfahl et al. (2003). Here one needs to be cautious that, although the numbers of LMXBs in the Galactic disk with measured orbital periods and accretion rates have been substantially increased (51 and 45 respectively) since then, this comparison is still severely subject to small number statistics as well as observational selection effects, e.g., luminous sources are more likely to be observed. Since in this work several key procedures involved in the formation processes of I/LMXBs have been significantly upgraded, the accretion rate discrepancy, if really exists, strongly suggests that something is missing or needs to be modified when modeling the evolution of LMXBs.

A possible mechanism that may help solve the problem is that there are extra AML mechanisms in the LMXB evolution that are not taken into account. The calculated accretion rate distribution is peaked around $\lesssim 10^{-10} M_{\odot} \mathrm{yr}^{-1}$, implying that the mass transfer is driven by GR. There is similar situation in CVs, in which AML is thought to be dominated by MB and GR above and below the period gap, respectively. However, modeling CV evolution indicates that the AML rate below the period gap is $2.47( \pm 0.22)$ times the GR rate Knigge et al. 2011), suggesting the existence of some other AML mechanisms. Shao \& Li (2012a) considered several kinds of AML mechanisms including isotropic wind from NSs, outflows from the inner and outer Langrangian points, and the formation of a circumbinary disk. They found that only outflow from the outer Langrangian point (or a circumbinary disk) can account for the extra AML rate, provided that $15-45 \%$ of the transferred mass is lost from the binary. These consequential AML mechanisms can not only substantially enhance the mass accretion rates, but also influence the value of the bifurcation periods (e.g., Ma \& 
\begin{tabular}{l|l|l}
\hline Li & $2009)$.
\end{tabular}

Another possible origin of the accretion rate discrepancy is that the calculated secular mass transfer rate may not be identical to the accretion rate derived based on short time (no more than $\sim 40$ years) observations. For example, X-ray irradiation of the donor star may driven mass transfer cycles in LMXBs (Podsiadlowski 1991; Büning \& Ritter 2004; Benvenuto et al. 2015). In this picture, once mass transfer begins, the accretion luminosity enhances the irradiation flux on the donor. Thermal expansion of the donor subsequently triggers an increasing mass transfer rate (this stage is denoted as high state). The donor evolves to a new thermal equilibrium state on Kelvin-Helmholtz timescale. When the donor nearly reaches the equilibrium state, its expansion rate decreases, then the mass transfer rate drops, and so does the irradiation flux. The donor starts to contract, and its intrinsic luminosity increases. This reduces the mass transfer to a quite low rate (denoted as low state). Hence the LMXB undergoes cyclic mass transfer alternating between high and low states. Although spending most of their evolutionary time in low state, they are more likely to be detected in high state, in which the mass transfer rates can be significantly larger than the average ones.

In both cases mentioned above the X-ray lifetime is shortened, which can increase the birthrates of LMXBs derived from the observed number in the Galactic disk. However, the detailed processes related to either extra AML loss or cyclic mass transfer are rather uncertain and depend on poorly known input parameters. Numerical calculations show that irradiation in LMXBs may boost the mass transfer rates by tens of times (Büning \& Ritter 2004; Benvenuto et al. 2015). Furthermore, Ritter (2008) argued that the mass transfer cycles are not likely to work for transient sources with an unstable disk, because irradiation cannot be sustained in a long quiescent stage. To illustrate the effect of irradiation in a simplified way, we arbitrarily increase the mass transfer rates of persistent LMXBs by a factor of $\Gamma$, which is randomly distributed between 1 and 30. The corresponding evolutionary time is then reduced by the same factor. The resultant distribution is plotted in Fig. 11, and we see that it seems to better match the observational data, especially for the luminous sources. The values of $M_{\mathrm{KS}}$ decreases to be 3.8 and 2.9 correspondingly.

\subsection{Orbital period distribution of BMSPs}

Similar as in Paper I, we follow the evolution of LMXBs to the formation of binary MSPs, and plot their orbital period distributions with the black lines in Fig. 11. In most cases the mass transfer terminates when the donor star loses its hydrogen-rich envelope and

becomes a He or CO WD. For converging LMXBs, we do not follow the evolution when the 
systems nearly reach the minimum periods. They might evolve to be black widow systems (e.g., Chen et al. 2013; Benvenuto et al. 2014). At the end of each evolutionary sequence, we know the final orbital period and the secondary mass. By summing the calculated sequences, we obtain the orbital period distributions of BMSPs. We assume that the lifetimes of all BMSPs are $10^{9} \mathrm{yr}$, so their numbers in each bin of the orbital period depends on their birthrates.

In Fig. 12 we also plot the observed orbital period distributions of binary pulsars in the red lines. The upper and lower panels correspond to the initial NS mass of $1.0 M_{\odot}$ and $1.8 M_{\odot}$, respectively. Depending on the amount of the accreted mass, the NSs may be fully or partially recycled. It is well known that accretion of $\sim 0.1 M_{\odot}$ material is sufficient to spin up a NS to millisecond period (e.g., Tauris et al. 2012), so we distinguish BMSPs from other partially recycled pulsars using this criterion. In the left panel we show the theoretical and observational histograms for both fully and partially recycled NSs, that is, those with any accreted mass $\Delta M_{\mathrm{NS}} \geq 0$, and those with measured spin periods $P_{\mathrm{s}} \leq 1 \mathrm{~s}$, respectively. In the right panel we are confined with BMSPs, that is, those with $\Delta M_{\mathrm{NS}} \geq 0.1 M_{\odot}$, and those with $P_{\mathrm{s}} \leq 10 \mathrm{~ms}$ (data from the ATNF pulsar catalogue ${ }^{6}$ Manchester et al. 2005).

The orbital periods of Galactic binary pulsars extend to $\sim 1000$ days, in agreement with our calculated results with $\Delta M_{\mathrm{NS}} \geq 0$, while those of BMSPs tend to have systematically small values. The known BMSPs have orbital periods up to 200 days, longer than predicted if $M_{\mathrm{NS}}=1 M_{\odot}$ but likely compatible with that if $M_{\mathrm{NS}}=1.8 M_{\odot}$. However, our calculated distributions show a shortage of BMSPs with $P_{\text {orb }} \sim 0.1-10$ day, which conflicts with observations (see also Pfahl et al. 2003). Figure 13 presents the CDFs for the orbital periods of binary pulsars with $P_{\text {orb }}>0.1$ day. Similar as in Fig. 12, the left and right panels correspond to all binary pulsars and BMSPs, respectively. The line styles are same as in Fig. 10. There are 115 and 77 binary pulsars in the Galactic disk with $P_{\mathrm{s}}<1 \mathrm{~s}$ and $P_{\mathrm{s}}<10$ ms in the ATNF pulsar catalogue, respectively. At a significance level $\alpha=0.05, M_{\mathrm{KS}}^{\mathrm{cr}} \simeq 1.4$. In the left panel, $M_{\mathrm{KS}} \simeq 2.5$ and 3.9 for $M_{N S}=1.0 M_{\odot}$ and $1.8 M_{\odot}$, respectively. In the right panel, $M_{\mathrm{KS}} \simeq 1.7$ and 2.1 for $M_{N S}=1.0 M_{\odot}$ and $1.8 M_{\odot}$, respectively. In both cases the $M_{\mathrm{KS}}$ values are larger than $M_{\mathrm{KS}}^{\mathrm{cr}}$ at $P_{\mathrm{orb}} \sim 0.1-10$ days.

The rarity of the predicted narrow BMSPs is likely to be related to the accretion rate discrepancy discussed before, as the orbital period evolution largely depends on the mass transfer process. It is caused by the fact that in our (and almost all previous) calculations RLOF is mainly driven by nuclear evolution of the donor or MB, so that the bifurcation period is around $0.3-1$ day (Pylyser \& Savonije 1988, 1989; Ma \& Li 2009). For binaries

\footnotetext{
${ }^{6}$ http://www.atnf.csiro.au/research/pulsar/psrcat/
} 
with initial orbital periods around it, mass transfer will finally lead to either wider or narrower orbits. Only with the initial parameters in a very small parameter space can LMXBs evolve into this orbital period range. It seems that neither changing the MB law nor considering the feedback of X-ray irradiation can solve this problem in a satisfactory manner (see also Istrate et al. 2014, for a relevant discussion). Similar problems also exist for the formation of UCXBs. It was found that with the standard MB law, only a very small range of initial parameters are allowed for the binaries to evolve to UCXBs within Hubble time (Podsiadlowski et al. 2002; van der Sluys et al. 2005a); if a saturated MB law is adopted, no UCXBs can be formed at all (van der Sluys et al. 2005b). This makes it impossible to account for the relatively large number of observed UCXBs. All these facts point to some missing (or misunderstood) AML mechanisms in the LMXB evolution, which will be investigated in a future work.

\section{Summary}

In this work, we incorporate detailed evolutionary calculations with BPS to explore the formation and evolutionary tracks of I/LMXBs in the Galaxy. By this way, we can trace the evolution of the I/LMXBs from their incipient stage to the descendant binary pulsars. Here we summarize the results as follows.

1. The incipient I/LMXBs contain NSs formed by both core-collapse and electroncapture SNe. Their orbital periods are distributed in the range of $\sim 0.2-100$ days and $\sim 0.2-1000$ days, respectively. The total birthrate of Galactic I/LMXB population is $\sim 9 \times 10^{-6}-3.4 \times 10^{-5} \mathrm{yr}^{-1}$ with a star formation rate of $5 M_{\odot} \mathrm{yr}^{-1}$.

2. Since the observability of I/LMXBs depends on the X-ray lifetimes and the stability of the accretion disks, Galactic LMXBs tend to be compact binaries with orbital periods $\lesssim$ 1 day and donor masses $M_{\mathrm{d}} \lesssim 0.3 M_{\odot}$. These features are consistent with those of observed LMXBs. However, the average mass transfer rate $\lesssim 10^{-10} M_{\odot} \mathrm{yr}^{-1}$ for persistent X-ray binaries is considerably lower than observed.

3. The orbital periods of BMSPs are predicted to extend from less than 1 day to hundreds of days, covering the majority of observed BMSPs. However, the number of BMSPs with $P_{\text {orb }} \sim 0.1-10$ day is severely underestimated.

4. The conflicts mentioned above imply that something is missing in the modeling of LMXBs, which is likely to be related to the (unknown) AML mechanisms.

This work was supported by the Natural Science Foundation of China under grant numbers 11133001, 11203009 and 11333004, and the Strategic Priority Research Program of 
CAS (under grant number XDB09000000).

\section{REFERENCES}

Bandyopadhyay, R. M., Shahbaz, T., Charles, P. A., \& Naylor, T. 1999, MNRAS, 306, 417

Belczynski, K., Kalogera, V., Rasio, F., Taam, R., Zezas, A., Bulik, T., Maccarone, T., \& Ivanova, N. 2008, ApJS, 174, 223

Benvenuto, O. G., De Vito, M. A., \& Horvath, J. E. 2014, ApJ, 786, L7

Benvenuto, O. G., De Vito, M. A., \& Horvath, J. E. 2015, ApJ, 798, 44

Bhattacharya, D., \& van den Heuvel, E. P. J. 1991, Phys. Rev., 203, 1

Büning, A., \& Ritter, H. 2004, A\&A, 423, 281

Casares, J., Charles, P., \& Kuulkers, E. 1998, ApJ, 493, L39

Chen, H.-L., Chen, X., Tauris, T. M., \& Han, Z. 2013, ApJ, 775, 27

Chen, W.-C., Li, X.-D., \& Xu, R.-X., 2011, A\&A, 530, 104

Coriat, M., Fender, R. P., \& Dubus, G. 2012, MNRAS, 424, 1991

D’Antona, F., Ventura, P., Burderi, L., Di Salvo, T., \& Lavagetto, G. et al. 2006, ApJ, 640, 950

de Kool, M. 1990, ApJ, 358, 189

Deloye C. J. 2008, in 40 YEARS OF PULSARS: Millisecond Pulsars, Magnetars and More. AIP Conference Proceedings, Eds. C. Bassa, Z. Wang, A. Cumming, \& V. M. Kaspi, Vol. 983, p. 501

de Mink, S. E., Pols, O. R., Langer, N., \& Izzard, R. G. 2009, A\&A, 507, L1

Dessart, L., Burrows, A., Ott, C. D., Livne, E., Yoon, S.-C., \& Langer, N. 2006, ApJ, 644, 1063

De Vito, M. A., \& Benvenuto, O. G. 2010, MNRAS, 401, 2552

Dewi, J. \& Tauris, T. 2000, A\&A, 360, 1043

Dubus, G., Lasota, J.-P., Hameury, J.-M., \& Charles, P. 1999, MNRAS, 303, 139 
Eggleton, P. P. 1971, MNRAS, 151, 351

Eggleton, P. P. 1972, MNRAS, 156, 361

Faulkner, J. 1971, ApJ, 170, L99

Feigelson, E. D., \& Jogesh Babu, G. 2012, Modern Statistical Methods for Astronomy, Cambridge University Press (Cambridge, UK)

Fryer, C., Belczynski, K., Wiktorowicz, G., Dominik, M., Kalogera, V., \& Holz, D. 2012, ApJ, 749, 91

Hjellming, M. S., \& Webbink, R. F. 1987, ApJ, 318, 794

Hobbs, G., Lorimer, D. R., Lyne, A. G., \& Kramer, M. 2005, MNRAS, 360, 974

Hurley, J. R., Pols, O. R., \& Tout, C. A. 2000, MNRAS, 315, 543

Hurley, J. R., Tout, C. A., \& Pols, O. R. 2002, MNRAS, 329, 897

Ivanova, N. et al. 2013, A\&AR, 21, 59

Istrate, A. G., Tauris, T. M., \& Langer, N. 2014, A\&A, 571, A45

Jia, K. \& Li, X.-D. 2014, ApJ, 791, 127

Jones, S., Hirschi, R., \& Nomoto, K. 2014, ApJ, 797, 83

Kalogera, V., \& Webbink, R. F. 1996, ApJ, 458, 301

Kiel, P. D., \& Hurley, J.R., 2006, MNRAS, 369, 1152

King, A. R. 1988, QJRAS, 29, 1

King, A. R., Kolb, U., \& Sienkiewicz, E. 1997, ApJ, 488, 89

King, A. R., \& Ritter, H. 1999, MNRAS, 309, 253

Knigge, C., Baraffe, I., \& Patterson, J. 2011, ApJS, 194, 28

Kolb, U., Davies, M. B., King, A., \& Ritter, H. 2000, MNRAS, 317, 438

Kroupa, P., Tout, C. A., \& Gilmore, G. 1993, MNRAS, 262, 545

Landau, L. D. \& Lifshitz, E. M. 1959, The Classical Theory of Fields (Oxford: Pergamon Press) 
Lasota, J.-P. 2001, New A Rev., 45, 449

Li, X.-D. 2002, ApJ, 564, 930

Li, X.-D. 2015, New A Rev., 64, 1

Lin, J., Rappaport, S., Podsiadlowski, P. et al. 2011, ApJ, 732, 70

Liu, Q. Z., van Paradijs, J., \& van den Heuvel, E. P. J. 2007, A\&A, 469, 807

Ma, B., \& Li, X.-D. 2009, ApJ, 691, 1611

Manchester, R. N., Hobbs, G. B., Teoh, A., \& Hobbs, M. 2005, AJ, 129, 1993

Nelson, C. A., \& Eggleton, P. P. 2001, ApJ, 552, 664

Orosz, J. A., \& Kuulkers, E. 1999, MNRAS, 305, 132

Packet, W. 1981, A\&A, 102, 17

Paczynski, B. 1976, In Structure and Evolution in Close Binary Systems. Proc. IAU Symp. 73, Eds., Eggleton, P. P., Mitton, S., \& Whealan, J. (Reidel, Dordrecht), p. 75

Pfahl, E., Rappaport, S., \& Podsiadlowski, P. 2003, ApJ, 597, 1036

Podsiadlowski, P. 1991, Nature, 350, 136

Podsiadlowski, Ph., \& Rappaport, S. 2000, ApJ, 529, 946

Podsiadlowski, Ph., Rappaport, S., \& Pfahl, E. D. 2002, ApJ, 565, 1107

Podsiadlowski, Ph., Rappaport, S., \& Han, Z., 2003, MNRAS, 341, 385

Patruno, A., \& Watts, A. L. 2012, arXiv:1206.2727

Poelarends, A. J. T., Herwig, F., Langer, N., \& Heger, A. 2008, ApJ, 675, 614

Pylyser, E., \& Savonije, G. J. 1988, A\&A, 191, 57

Pylyser, E., \& Savonije, G. J. 1989, A\&A, 208, 52

Rappaport, S., Joss, P. C., \& Verbunt, F. 1983, ApJ, 275, 713

Rappaport, S., Podsiadlowski, Ph., Joss, P. C., Di Stefano, R., \& Han, Z. 1995, MNRAS, 273,731 
Rawls, M. L., Orosz, J. A., McClintock, J. E., Torres, M. A. P., \& Bailyn, C. D. et al. 2011, ApJ, 730, 25

Revnivtsev, M., Postnov, K., Kuranov, A., \& Ritter, H. 2011, A\&A, 526A, 94

Ritter, H. 2008, New A Rev., 51, 869

Ritter, H., \& Kolb, U. 2003, A\&A, 404, 301

Robitaille, T. P., \& Whitney, B. A. 2010, ApJ, 710, L11

Shao, Y., \& Li, X.-D. 2012a, ApJ, 745, 165

Shao, Y., \& Li, X.-D. 2012b, ApJ, 756, 85 (Paper I)

Shao, Y., \& Li, X.-D. 2014, ApJ, 796, 37

Siess, L. 2006, A\&A, 448, 717

Smith, L. F., Biermann, P., \& Mezger, P. G. 1978, A\&A, 66, 65

Soberman, G. E., Phinney, E. S., \& van den Heuvel, E. P. J. 1997, A\&A, 327, 620

Tauris, T. M., \& Savonije, G. J. 1999, A\&A, 350, 928

Tauris, T. M., van den Heuvel, E. P. J., \& Savonije, G. J. 2000, ApJ, 530, L93

Tauris, T. M., \& van den Heuvel E. P. J., 2006, in Compact Stellar X-Ray Sources, Eds., W. H G. Lewin, M. van der Klis (Cambridge: Cambridge University Press), 623

Tauris, T. M., Langer, N., \& Kramer, M. 2012, MNRAS, 425, 1601

van den Heuvel, E. P. J., \& Taam, R. 1984, Nature, 309, 235

van der Sluys, M. V., Verbunt, F., \& Pols, O. R. 2005a, A\&A, 440, 973

van der Sluys, M. V., Verbunt, F., \& Pols, O. R. 2005b, A\&A, 431, 647

van Paradijs, J. 1996, ApJ, 464, L139

Verbunt, F. \& Zwaan, C. 1981, A\&A, 100, L7

Webbink, R. F., Rappaport, S., \& Savonije, G. J. 1983, ApJ, 270, 678

Webbink, R. F. 1984, ApJ, 277, 355

Woosley, S. E., \& Heger, A. 2015, submitted to ApJ (arXiv:1505.06712) 
Wu, Y. X., Yu, W., Li, T. P., Maccarone, T. J., \& Li, X.-D. 2010, ApJ, 718, 620

Xu, X.-J., \& Li, X.-D. 2010, ApJ, 716, 114

Zhu, C., Lü, G., Wang, Z., \& Wang, N. 2012, PASP, 124, 195 

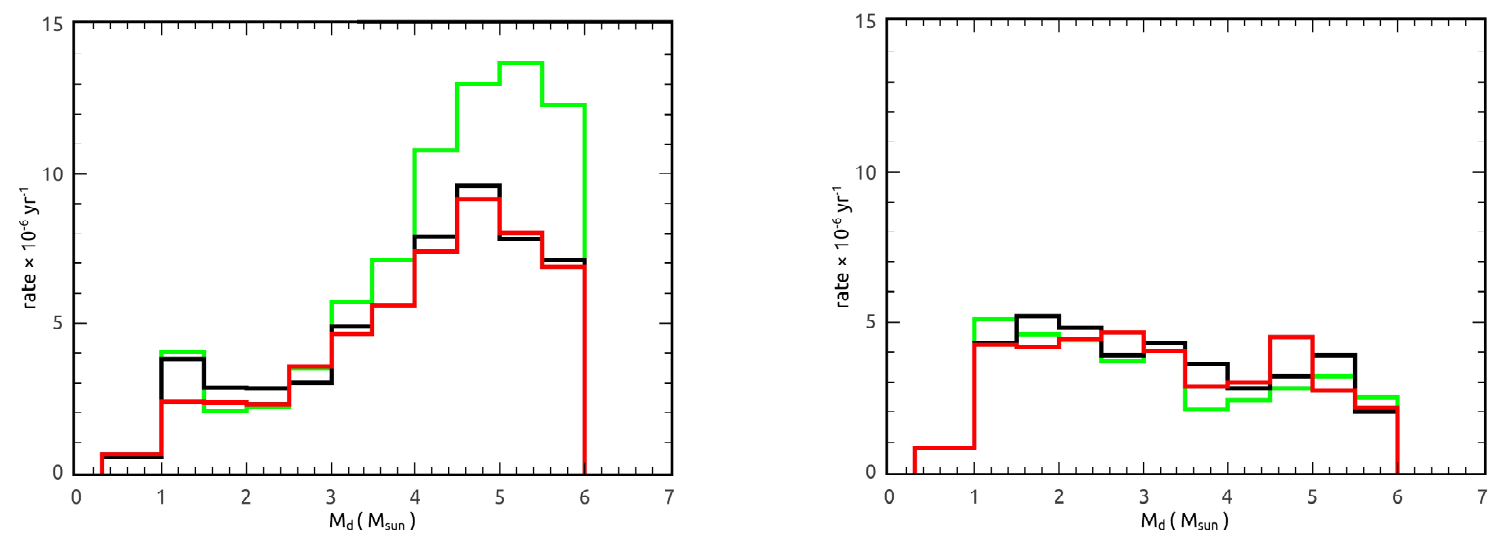

Fig. 1. - The birthrates of incipient I/LMXBs as a function of the donor mass $M_{\mathrm{d}}$ derived from the BPS calculations. Binaries containing NSs descended from core-collapse and electron-capture SNe are shown in the left and right panels, respectively. The green, red and black curves represent the results with the $q_{\mathrm{c}}$ values in Models I, II and III of Shao \& Li (2014), respectively.
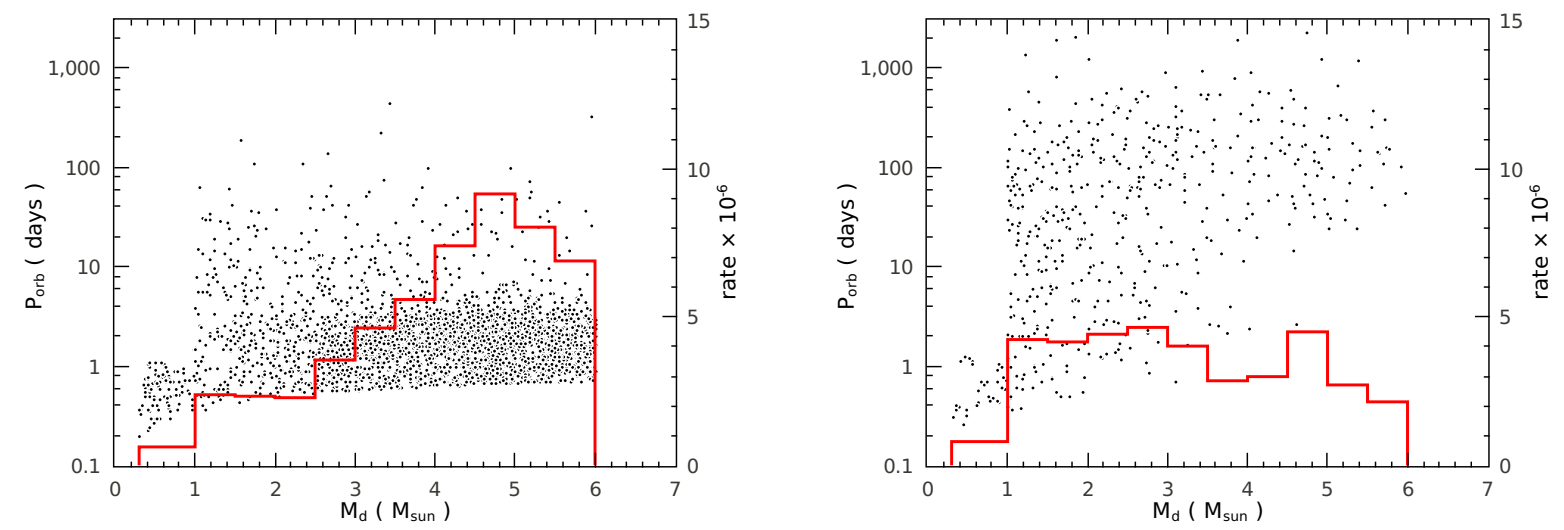

Fig. 2.- Distributions of incipient I/LMXBs in the plane of initial donor masses $M_{\mathrm{d}}$ and orbital periods $P_{\text {orb }}$. Binaries containing NSs descended from core-collapse and electroncapture SNe are shown in the left and right panels, respectively. The red curves reflect their birthrates (in Model II) as a function of $M_{\mathrm{d}}$. 

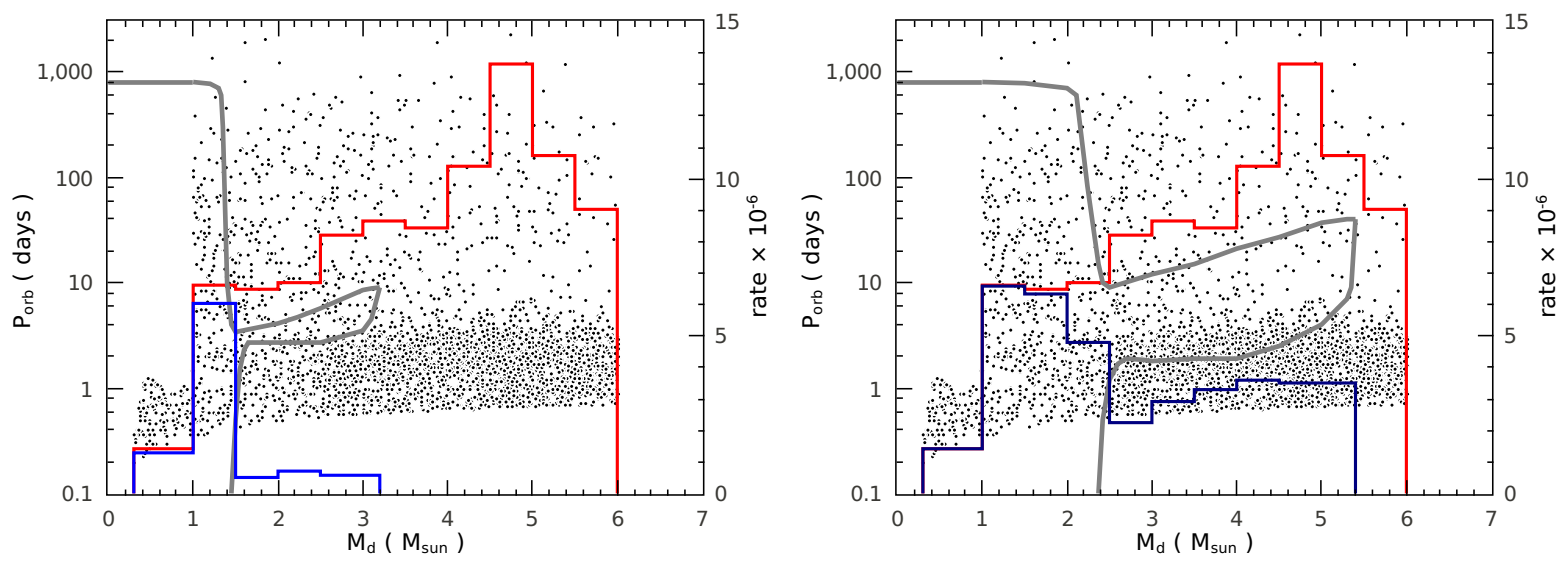

Fig. 3.- Distributions of incipient I/LMXBs (from both core-collapse and electron-capture SN channels) in the $M_{\mathrm{d}}-P_{\text {orb }}$ plane. The red curves reflect their birthrates as a function of $M_{\mathrm{d}}$, same as in Fig. 2. The grey curves confine the parameter space for stable mass transfer in X-ray binaries with an initial $1.0 M_{\odot}$ (left panel) and $1.8 M_{\odot}$ (right panel) $\mathrm{NS}$, and the birthrates for binaries within it are shown with the blue curves. 

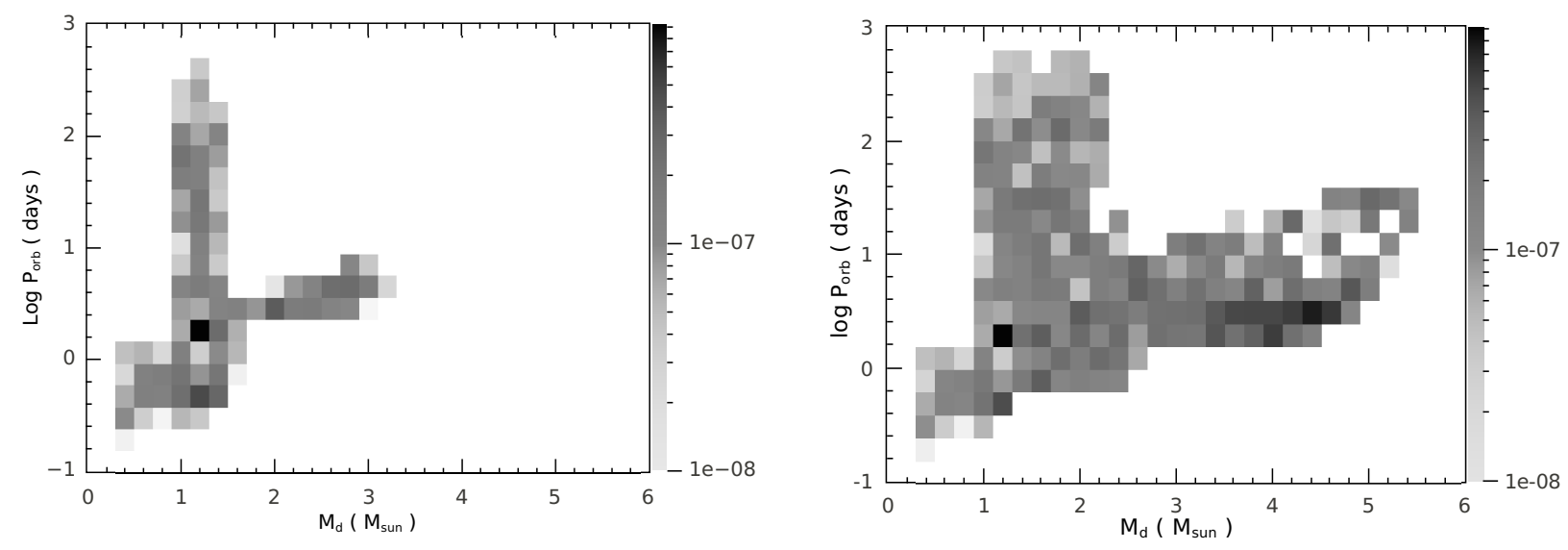

Fig. 4.- Distributions of of incipient I/LMXBs that have stable mass transfer in the $M_{\mathrm{d}}-$ $P_{\text {orb }}$ plane. 

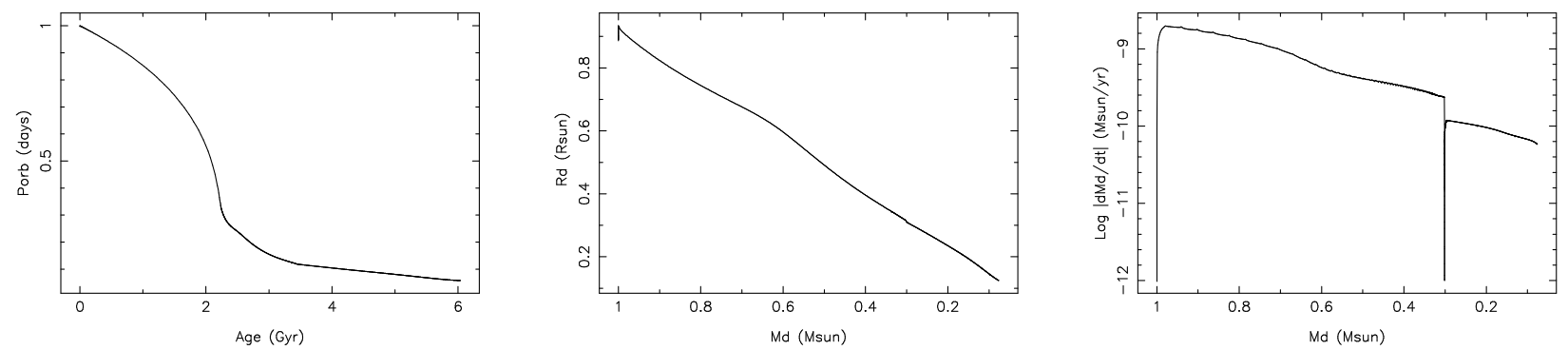

Fig. 5.- Evolutionary tracks of a LMXB with initial parameters of $M_{\mathrm{NS}}=1.8 M_{\odot}, M_{\mathrm{d}}=$ $1.0 M_{\odot}$ and $P_{\text {orb }}=1.0$ day. The left, middle, and right panels depict the orbital period $P_{\text {orb }}$ as a function of age, the donor radius $R_{\mathrm{d}}$ and the mass transfer rate $\left|\dot{M}_{\mathrm{d}}\right|$ as a function of the donor mass $M_{\mathrm{d}}$, respectively.
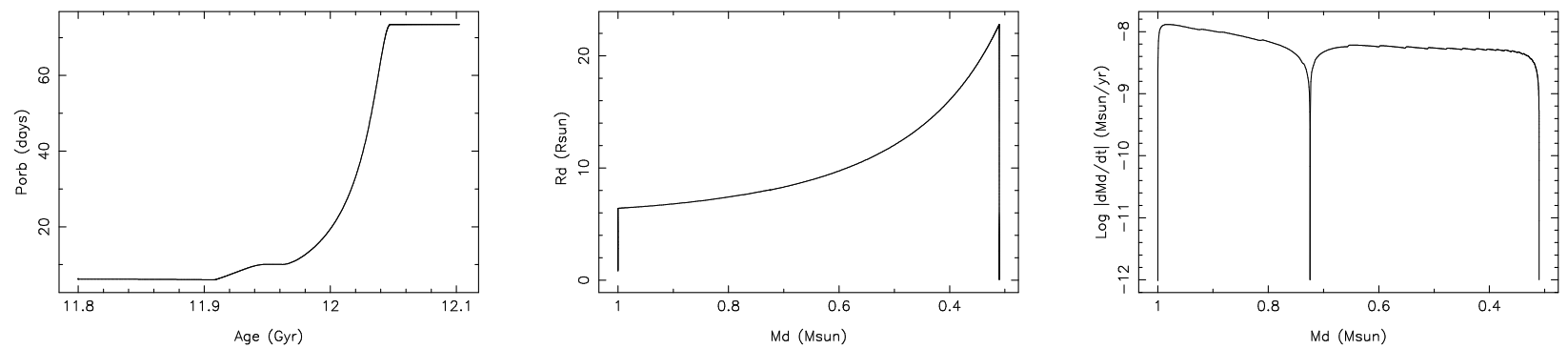

Fig. 6.- Similar to Fig. 5 but for an initial orbital period $P_{\text {orb }}=6.3$ days. 

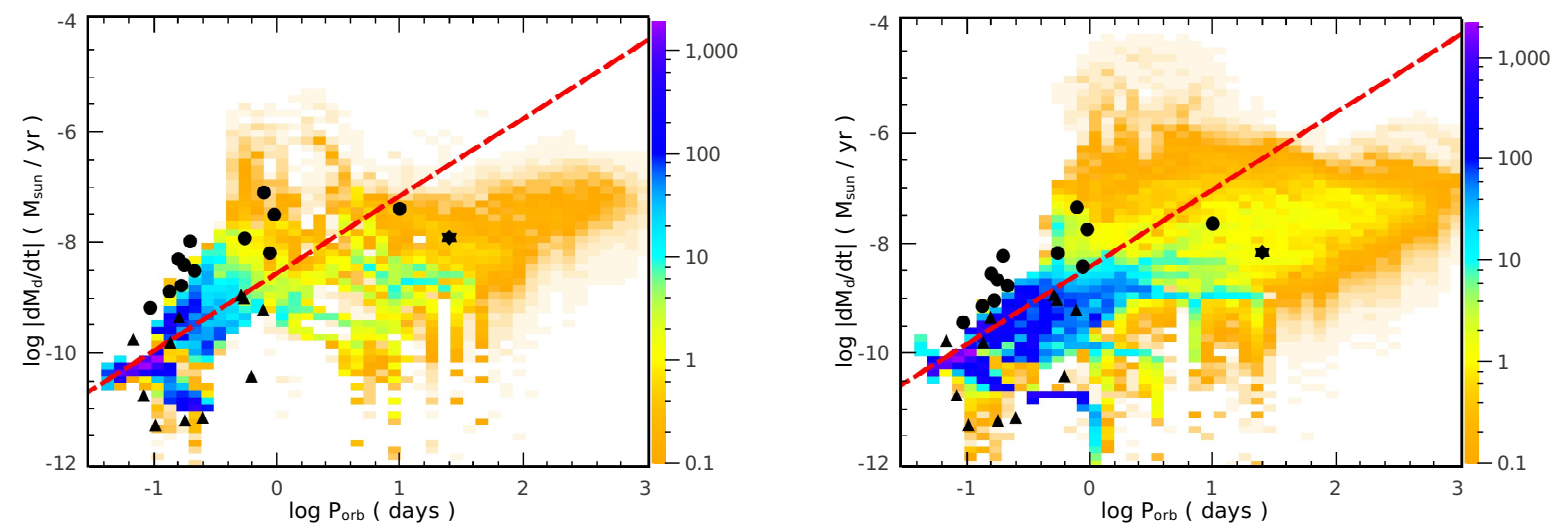

Fig. 7. - The orbital period and mass transfer rate distributions for I/LMXBs with a $1.0 M_{\odot}$ (left panel) and $1.8 M_{\odot}$ (right panel) NS. The colors represent the numbers of binaries in each pixel. The red dash line reflects the critical mass transfer rate for unstable accretion disks. The filled circles and triangles represent persistent and transient LMXBs in the Galactic disk, respectively. The filled star corresponds to GX $13+1$. 

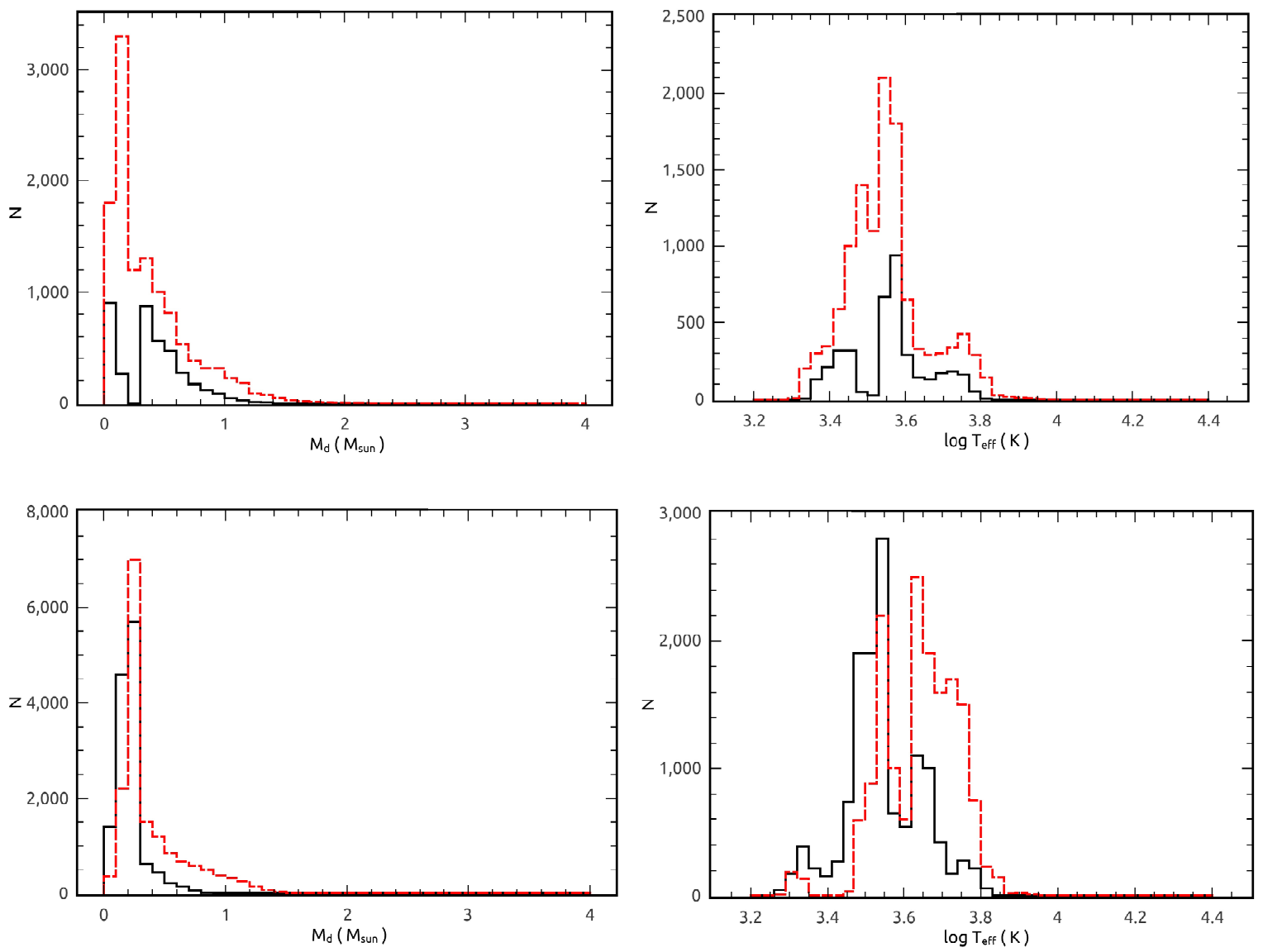

Fig. 8.- The calculated distributions of the donor mass (left panel) and effective temperature (right panel) in the I/LMXBs. The top and bottom panels show persistent and transient X-ray binaries, respectively. The black and red curves correspond to the initial NS mass of $1.0 M_{\odot}$ and $1.8 M_{\odot}$, respectively. 

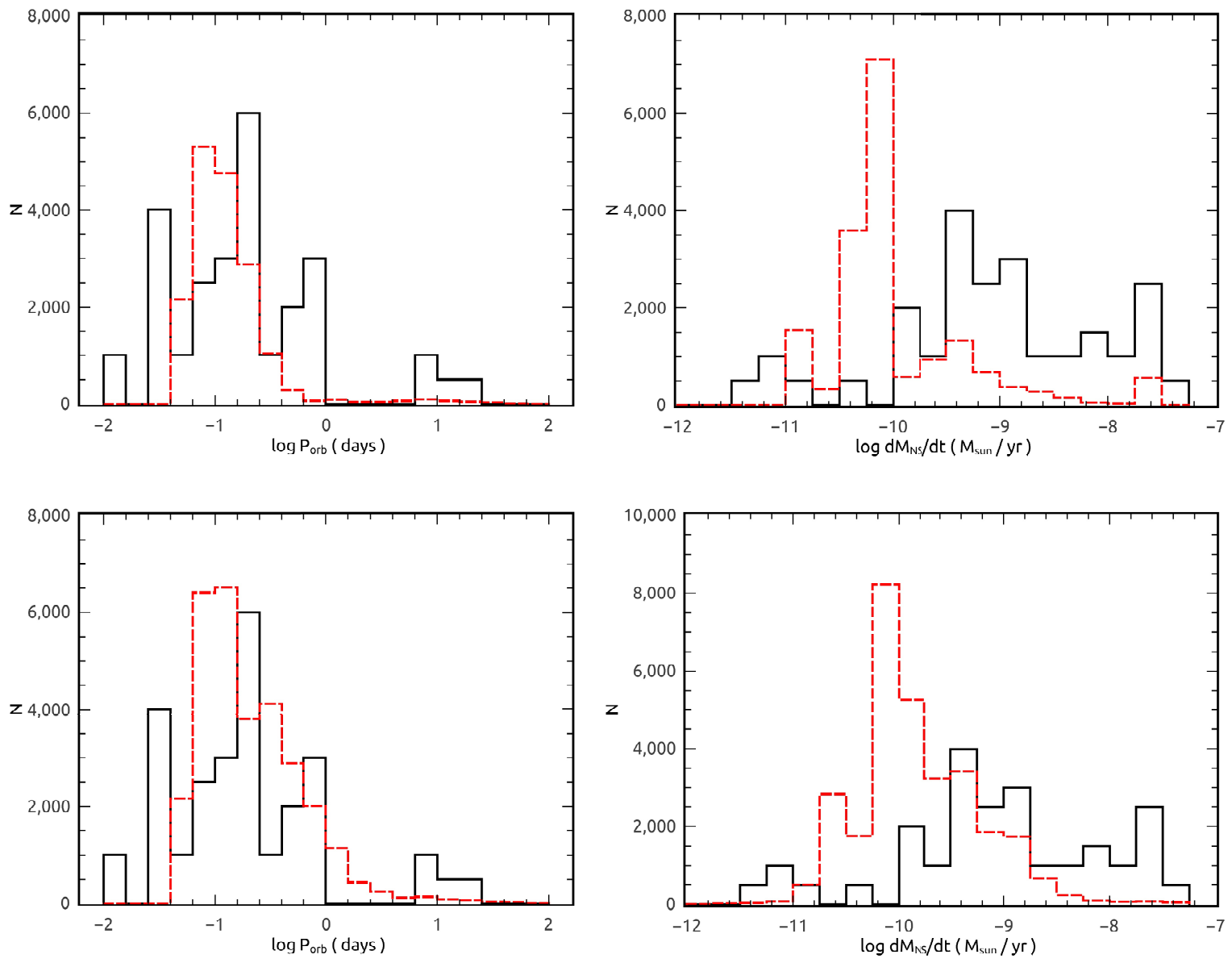

Fig. 9.- Comparison of the calculated orbital period (left panel) and accretion rate (right panel) distributions of Galactic I/LMXBs with observations, which are shown with the red and black curves, respectively. The top and bottom panels correspond to the initial NS mass of $1.0 M_{\odot}$ and $1.8 M_{\odot}$, respectively. 

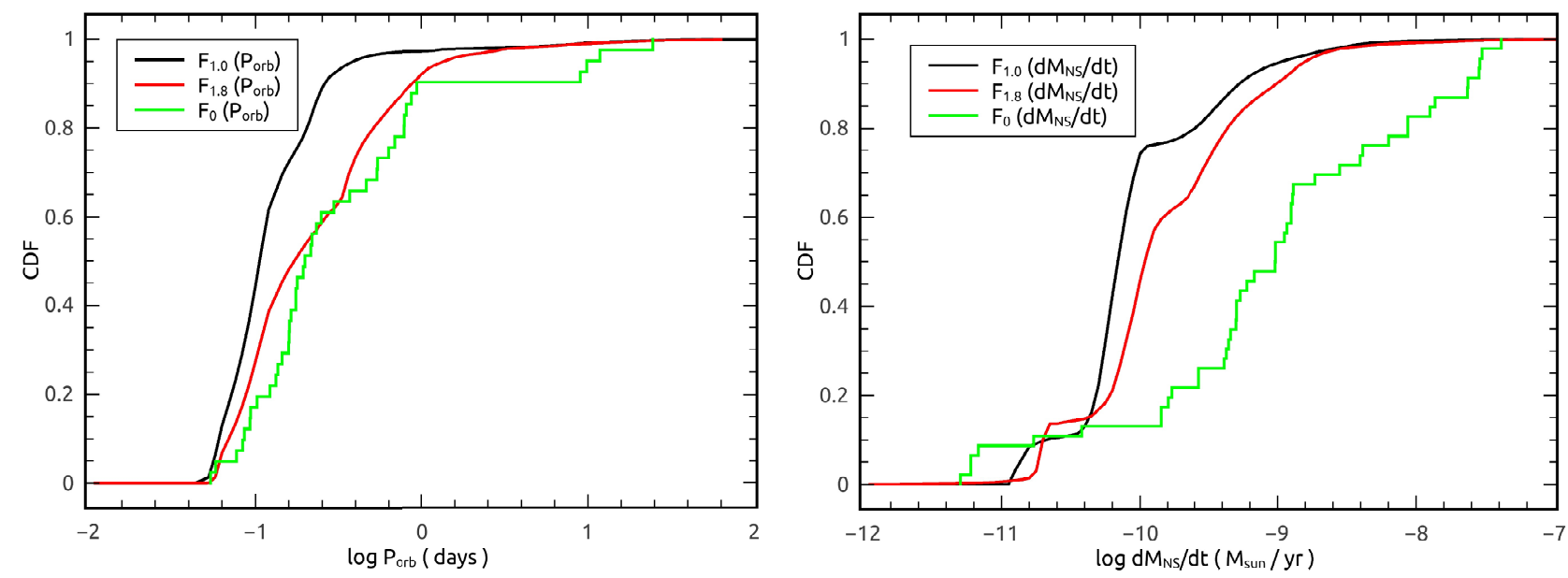

Fig. 10.- Cumulative distribution functions for the orbital period (left panel) and the accretion rate (right panel) of Galactic I/LMXBs. The green curve shows the observed distribution function. The black and red curves correspond to the calculated distribution functions with a $1.0 M_{\odot}$ and $1.8 M_{\odot} \mathrm{NS}$, respectively. 

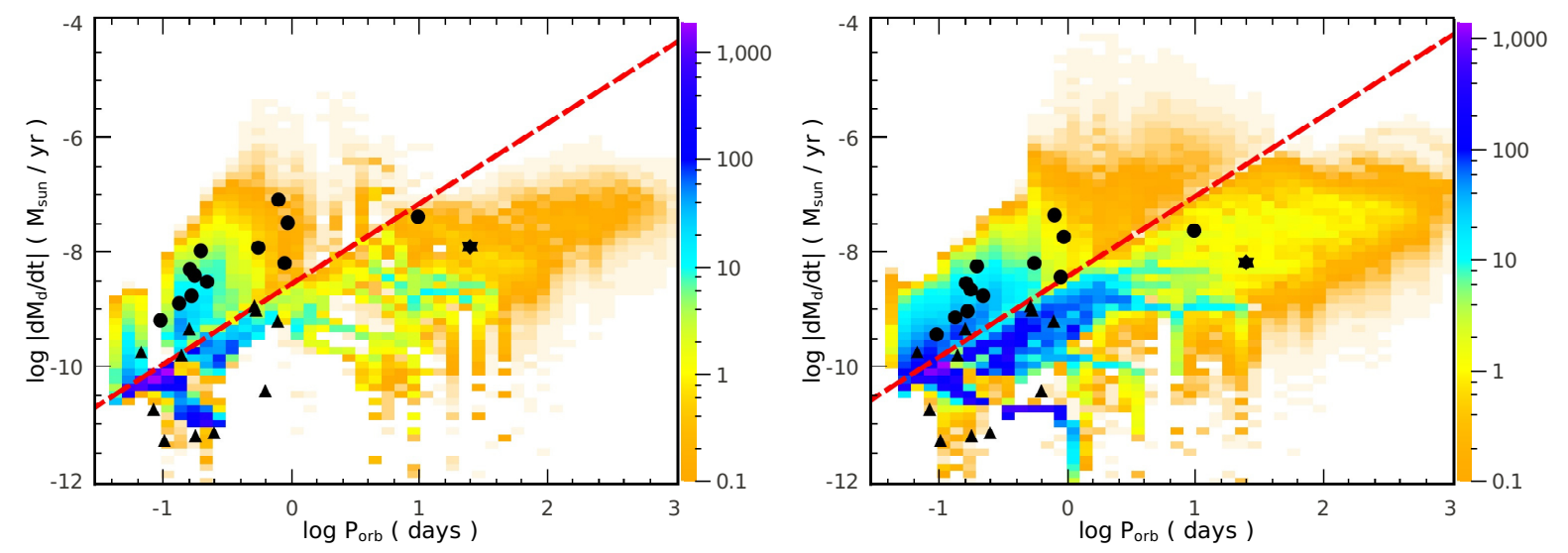

Fig. 11. - Similar as Fig. 7, but with the irradiation-induced mass transfer cycles taken into account. The mass transfer rates for persistent sources are arbitrarily increased by a factor between 1 and 30, while their lifetimes are decreased by the same factor. 

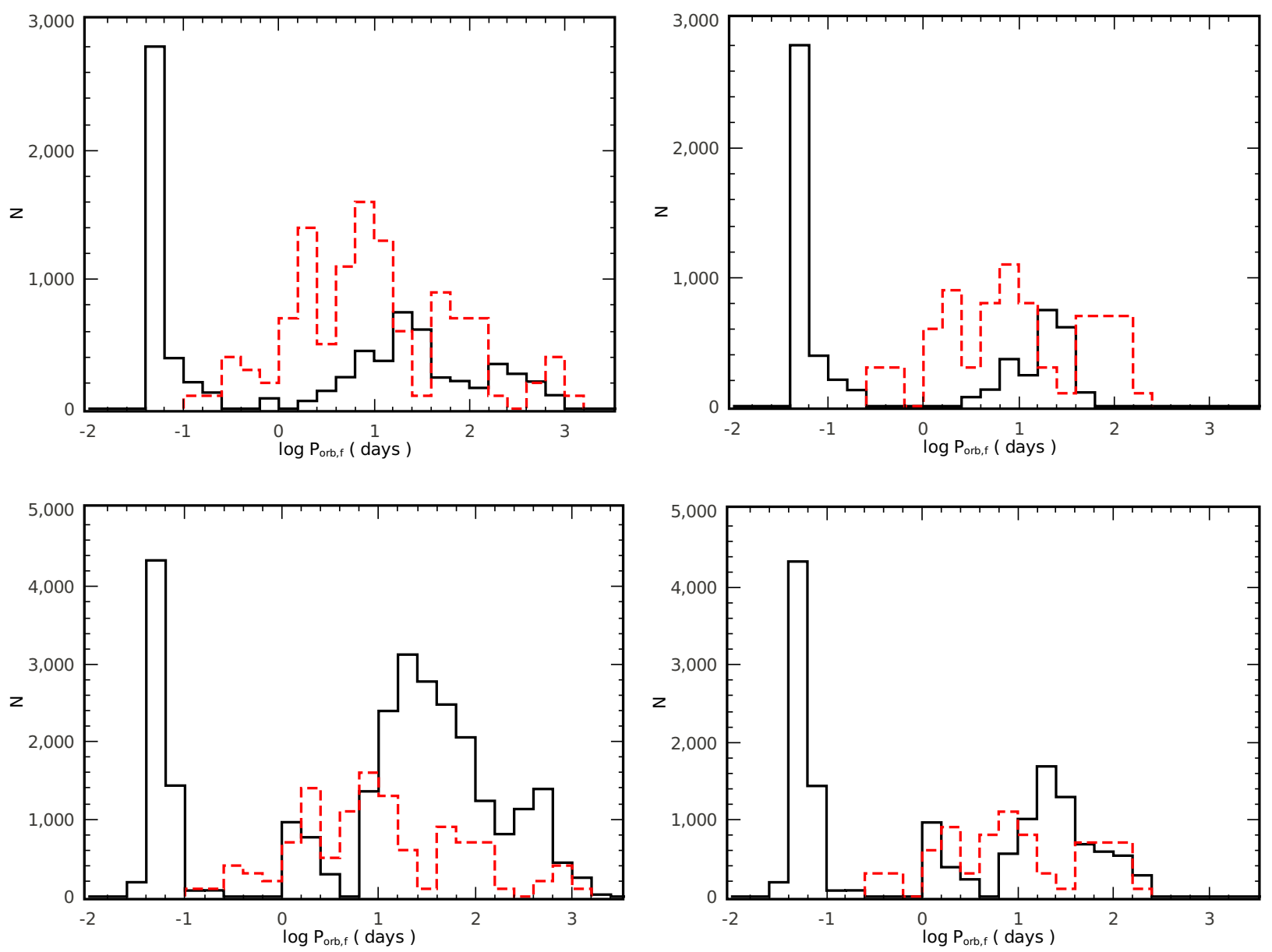

Fig. 12.- Comparison of the calculated and measured orbital period distributions of binary pulsars, which are shown with the black and red curves, respectively. The top and bottom panels correspond to the initial NS mass of $1.0 M_{\odot}$ and $1.8 M_{\odot}$, respectively. The left panel shows the calculated distributions of pulsars with any accreted mass $\Delta M_{\mathrm{NS}}>0$ and the observed distribution of binary pulsars with spin periods $P_{\mathrm{s}} \leq 1 \mathrm{~s}$. The right panel shows the calculated distributions of pulsars with accreted mass $\Delta M_{\mathrm{NS}} \geq 0.1 M_{\odot}$ and the observed distribution of binary pulsars with spin periods $P_{\mathrm{s}} \leq 10 \mathrm{~ms}$. 

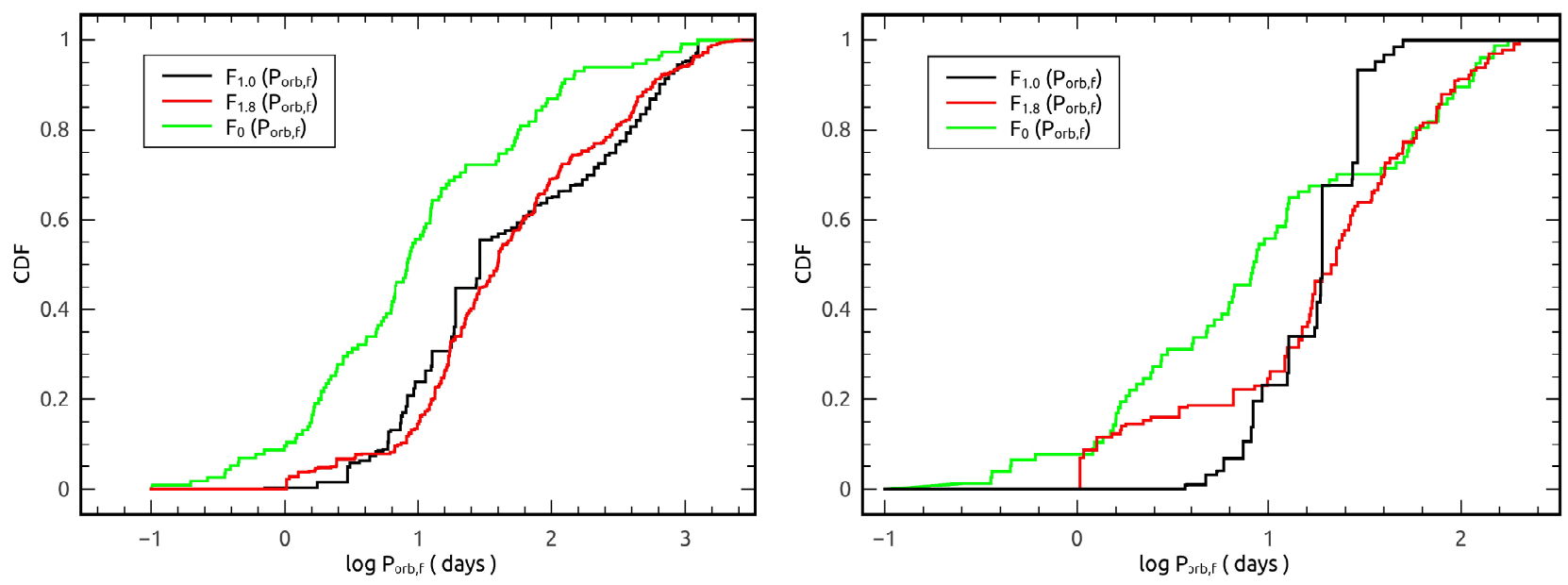

Fig. 13.- Cumulative distribution functions for the orbital periods of binary pulsars (left panel) and BMSPs (right panel). The line styles are same as in Fig. 11. 